\title{
Derivation of a Bogomol'ny Inequality in Five-Dimensional Kaluza-Klein Theory*
}

\author{
Joohan Lee and Rafael D. Sorkin \\ Physics Department, Syracuse University, Syracuse, NY 13244-1130, USA
}

\begin{abstract}
We prove an inequality bounding the mass from below by the electromagnetic charge in five-dimensional Kaluza-Klein theory without assuming the existence of global $U(1)$ symmetry.
\end{abstract}

\section{Introduction}

Since Witten [1] discovered a simple proof of the positive energy theorem [2] in general relativity, various generalizations [3] have been made using similar methods. These results have important physical consequences for the stability of Minkowski spacetime. Recently there have been attempts $[4,5]$ to generalize this result to higher dimensional theories of Kaluza--Klein type (for a review on this subject see Ref. [6] and references therein).

In those theories, unlike in general relativity, there exist globally regular, asymptotically vacuum, static solutions, besides the vacuum solution, which are stable. These solutions have non-trivial topology even in the neighborhood of spatial infinity, and it would be reasonable to assume that this non-localizable topological non-triviality prevents them from decaying into a configuration belonging to the topological sector of the true vacuum (they are topological solitons). In any case to determine if a given solution is stable we need to know at least whether it is the minimum energy solution in its topological sector.

In this paper we consider five-dimensional Kaluza-Klein theory and prove an inequality bounding the mass from below by the electromagnetic charge without assuming the existence of an exact internal Killing symmetry. This relaxation of exact internal symmetry is necessary if we wish to allow non- $U(1)$-symmetric perturbations. The inequality we will derive turns out to be the same as the one formulated by Gibbons and Perry [5] using a supersymmetric argument. In the course of the derivation we will put special emphasis on solutions with non-zero magnetic charge. Examples of particular interest are the multimonopole solutions [7]. In each asymptotic topological sector, we show that these solutions saturate the inequality.

* Supported in part by NSF-Grant No. PHY-8318350 
The organization of this paper is as follows. In Sect. 2 we briefly explain five-dimensional Kaluza-Klein theory in a geometrical way. At least this will fix our notation. Section 3 is the main part of the paper which contains the derivation of the inequality in the presence of non-zero magnetic charge. In particular how topological charge (which we did not have in four dimensions) is incorporated into the inequality is explained. If spacetime is four-dimensional, then topological charge is not present, and our method yields a particularly compact derivation of the usual positivity theorem. To define conserved quantities in Sect. 3 we use a background connection $\stackrel{\circ}{\nabla}_{a}$. Some subtleties involved in extending $\stackrel{\circ}{\nabla}_{a}$ to spinor fields are discussed in the appendix. Finally in Sect. 4 we summarize the result obtained and discuss some unsolved problems.

\section{Five-Dimensional Kaluza-Klein Theory}

The most naive Kaluza-Klein theory unifies the electromagnetic and gravitational fields by considering a metric $g_{a b}$ of Lorentzian signature on a five-dimensional manifold. This five-dimensional spacetime is assumed to have a $U(1)$ symmetry generated by a spacelike vector field $k^{a}$ of constant norm $\lambda_{\infty}$, where this constant is regarded as a constant of the theory. By $U(1)$ symmetry we mean that the spacetime has a principal $U(1)$ bundle structure such that the structure group $U(1)$ is also an isometry group. We interpret the fiber, which is topologically a circle, as an extra spatial dimension at each point of an effective four-dimensional spacetime represented by the base manifold. In order to make this interpretation consistent with our observations the size of the internal circle has to be very small.

Let us recall how this five-dimensional metric joins the electromagnetic and gravitational fields together. From the metric $g_{a b}$, using the fact that it is Lie derived by $k^{a}$ (meaning it is independent of the internal coordinate), we can construct two horizontal tensor fields which are independent of the internal coordinate: a symmetric tensor field $h_{a b}:=g_{a b}-\lambda_{\infty}^{2} A_{a} A_{b}$ and an antisymmetric tensor field $F_{a b}:=\nabla_{a} A_{b}-\nabla_{b} A_{a}$, where $A_{a}=\lambda_{\infty}^{-2} g_{a b} k^{b}$. (A tensor field is called horizontal if it is zero when contracted with $k^{a}$.) It is easy to verify that $h_{a b} k^{b}=0, F_{a b} k^{b}=0$. Because any horizontal tensor field which is Lie-derived by $k^{a}$ can be identified with a tensor field on the base manifold, the metric $g_{a b}$ defines for us two tensor fields on the effective four-dimensional spacetime.

Let us choose the Einstein-Hilbert action and express it in terms of $h_{a b}$ and $F_{a b}$. The result is:

$$
(1 / 2 k) \int{ }^{5} R d^{5} v=\left(2 \pi \lambda_{\infty} / k\right) \int\left[(1 / 2){ }^{4} R-\left(\lambda_{\infty}^{2} / 8\right) F_{a b} F^{a b}\right] d^{4} v,
$$

where we used the metric $h_{a b}$ in forming the Ricci scalar ${ }^{4} R$ and the invariant volume element $d^{4} v$, and in raising the indices of $F_{a b}$. We immediately recognize (2.1) as the action for the Einstein-Maxwell system without source if we identify $k / 2 \pi \lambda_{\infty}$ with $\kappa=8 \pi G, F_{a b}$ with the Maxwell field and $h_{a b}$ with the four-dimensional metric. It is in this sense that the most naive Kaluza-Klein theory unifies the electromagnetic and gravitational fields. Although we needed to fix the norm of $k^{a}$ to obtain the Einstein-Maxwell equation in the effective four-dimensional spacetime, it is artificial, from the five-dimensional point of view, to fix one 
component of the five-metric as a kinematical structure. For instance, since we do not vary the internal-internal component of the metric, $g_{a b} k^{a} k^{b}$ (it being a constant of the theory), the corresponding component of the Einstein tensor, $G_{a b} k^{a} k^{b}$, is not constrained at all.

A less artificial version of the Kaluza-Klein theory, which is the most popular one, does not fix a priori the norm of the $U(1)$ Killing vector $k^{a}$. The norm of $k^{a}$ is allowed to vary and turned into a dynamical field with the boundary condition that it approaches the constant value $\lambda_{\infty}$ as we go to infinity. Then the five-dimensional field equation in this theory becomes exactly the vacuum Einstein equation.

To see what kind of fields and their dynamics this theory describes let us express the Einstein-Hilbert action in terms of tensor fields naturally defined on the base manifold. If we define $\lambda:=\left(g_{a b} k^{a} k^{b}\right)^{1 / 2}, h_{a b}:=g_{a b}-\lambda^{2} A_{a} A_{b}, F_{a b}:=\nabla_{a} A_{b}-\nabla_{b} A_{a}$ with $A_{a}=\lambda^{-2} g_{a b} k^{b}$ then, as before, $\lambda, h_{a b}$, and $F_{a b}$ can be identified with four-dimensional tensor fields. In terms of these tensor fields the action is:

$$
(2 \pi / k) \int\left[(\lambda / 2){ }^{4} R-\left(\lambda^{3} / 8\right) F_{a b} F^{a b}-D_{a} D^{a} \lambda\right] d^{4} v .
$$

As before $h_{a b}$ was used for ${ }^{4} R, d^{4} v$, and index raising, and $D_{a}$ is the covariant derivative operator such that $D_{a} h_{b c}=0$. We can make this expression look more familiar by performing the following conformal transformation:

$$
h_{a b} \rightarrow \tilde{h}_{a b}:=\Omega^{2} h_{a b}, \quad \Omega^{2}=\left(\lambda / \lambda_{\infty}\right) .
$$

Then (2.2) becomes:

$$
(1 / 2 \kappa) \int\left[{ }^{4} \tilde{R}-(1 / 4) \lambda^{2} \Omega^{2} \tilde{F}_{a b} \tilde{F}^{a b}+\tilde{D}_{a} \tilde{D}^{a} \ln \lambda-(3 / 2)\left(\tilde{D}_{a} \ln \lambda\right)\left(\tilde{D}^{a} \ln \lambda\right)\right] d^{4} \tilde{v},
$$

where $\kappa:=k / 2 \pi \lambda_{\infty}$ as before and we have used $\tilde{h}_{a b}$ as a metric for construction and index raising of objects with tilde's. If we drop the total divergence term, this is the action for general relativity with an unusual coupling between two matter fields, namely a scalar field and the Maxwell field. Some interesting solitonic solutions have been found in this theory [7].

However, it is believed that imposing an exact $U(1)$ symmetry is still artificial, and one should allow the higher dimensional metric all of its degrees of freedom, hoping to recover the $U(1)$ symmetry as a dynamical low energy phenomenon. If we adopt this point of view we should not impose $U(1)$ symmetry at a kinematical level. In particular, transition to a non-symmetric configuration should not be excluded a priori as a possible mode of instability of the monopole or any other $U(1)$-symmetric soliton ${ }^{1}$.

Let us discuss our choice of the boundary conditions for this generalized version of the Kaluza-Klein theory. These boundary conditions will be assumed in the second part of the next section. In an asymptotically flat spacetime in four dimensions it is natural to choose a flat metric as the background structure to specify the boundary conditions necessary for various discussions which involve

To first order, stability against $U(1)$-symmetric perturbation implies stability against arbitrary ones, but this implication fails for finite perturbations 
globally defined physical quantities such as total energy-momentum, angular momentum and so on. However in the theory we are considering, it may not be possible to choose a flat metric even near spatial infinity because of a topological obstruction. The most interesting examples are solutions having non-zero magnetic charge. In such a case, even the background structure is subject to the topological restriction. The best we can do is to choose a background metric which, in some neighborhood of spatial infinity, has an exact $U(1)$ Killing vector $k^{a}$ of constant norm $\lambda_{\infty}$ and whose quotient metric is flat. In addition, we can impose that the metric is ultra-static and spherically symmetric near infinity. Roughly speaking, it is the metric which is as flat and as symmetric as possible in the given topological sector. One trivial consequence of this choice is that the total electric charge of the background is zero because electric field on a static slice vanishes. The total four-momentum is also zero. As would be expected the background has no dynamical feature. However, it may have a non-vanishing magnetic charge which is topological in origin and is determined by what topological sector it belongs to. With this background metric $\stackrel{\circ}{g}_{a b}$, our boundary condition is that any physical metric $g_{a b}$ should satisfy $g_{a b}-\stackrel{\circ}{g}_{a b}=0(1 / r)$.

\section{A Bogomolny Inequality}

In this section we prove an inequality bounding the mass from below by the electromagnetic charge without assuming the existence of an exact $U(1)$ symmetry. For this, we will use a spinorial method, which, in particular, requires that the spacetime in question has an appropriate spinor structure. This section is divided into two parts. In the first part we will show that the integral of certain spinor expression over a three-dimensional closed spacelike surface can be made less than or equal to zero if the spinor extends to one satisfying Witten's equation. For this, the standard method in four-dimensions goes through without any modification. Therefore we just present well-known results in a convenient way. In the second part we interpret the integral of the spinor expression at spatial infinity in terms of the 4-momentum and the electromagnetic charges, obtain thereby a non-zero minimum for the mass. This part will be done in some detail because the proof has been given in detail in the literature [4] only for the case where there exists an exact $U(1)$ symmetry.

a) First Part of Proof. Let $g_{a b}$ be a globally regular solution to the fivedimensional Einstein equation $G_{a b}=0$ satisfying the boundary conditions for the five-dimensional Kaluza-Klein theory, and consider the five-dimensional version of the spinor expression introduced by Witten (generalized by Nester):

$$
W^{a b}:=\operatorname{Im}\left(\bar{\psi} \gamma^{a b c} \nabla_{c} \psi\right)
$$

where $\gamma^{a b c}:=(1 / 6)\left(\gamma^{a} \gamma^{b} \gamma^{c}+\right.$ even permutations - odd permutations $)$ with $\gamma^{a}$ satisfying $\gamma^{a} \gamma^{b}+\gamma^{b} \gamma^{a}=2 g^{a b} I, \bar{\psi}$ is the Dirac-adjoint of the spinor $\psi$ (we choose the Dirac-adjoint so that $\bar{\gamma}^{a}=-\gamma^{a}$ ) and $\nabla_{a}$ is the torsion free covariant derivative operator of the metric $g_{a b}$ extended to spinor fields in the standard way. The symbol Im stands for taking the imaginary part and, for simplicity, we drop spinor indices. 
To show that the integral of $W^{a b}$ over a three-dimensional closed surface can be made less than or equal to zero, we need the following identity:

$$
\nabla_{b} W^{a b}=(1 / 2) G_{b}^{a} \xi^{b}+\operatorname{Im}\left(\nabla_{b} \bar{\psi} \gamma^{a b c} \nabla_{c} \psi\right)
$$

where $\xi^{b}=\operatorname{Im}\left(\bar{\psi} \gamma^{b} \gamma\right)$. This identity can be derived fairly easily using the following properties of $\nabla_{a}$ :

$$
\nabla_{a} \gamma^{b}=0, \quad\left(\nabla_{a} \nabla_{b}-\nabla_{b} \nabla_{a}\right) \psi=(1 / 4) R_{a b c d} \gamma^{c d} \psi,
$$

where $R_{a b c d}$ is the Riemann tensor of the metric $g_{a b}$, and $\gamma^{a b}=(1 / 2)\left(\gamma^{a} \gamma^{b}-\gamma^{b} \gamma^{a}\right)$. Also we use a Clifford algebra identity which looks more complicated than it really is. Because we will use that identity several times, we give it here in fully written out form:

$$
\begin{aligned}
\gamma^{a b c} \gamma^{m n}= & g^{c m} g^{b n} \gamma^{a}-g^{c n} g^{b m} \gamma^{a}+g^{b m} g^{a n} \gamma^{c}-g^{b n} g^{a m} \gamma^{c}+g^{a m} g^{c n} \gamma^{b}-g^{a n} g^{c m} \gamma^{b} \\
& +g^{c m} \gamma^{a b n}-g^{c n} \gamma^{a b m}+g^{b m} \gamma^{c a n}-g^{b n} \gamma^{c a m}+g^{a m} \gamma^{b c n}-g^{a n} \gamma^{b c m}+\gamma^{a b c m n}
\end{aligned}
$$

Among all these, terms containing a product of three $\gamma$ 's do not contribute in any of our calculations because $\left(\bar{\psi} \gamma^{a b c} \psi\right)$ is real. The last piece, an antisymmetric product of five $\gamma$ 's does contribute in general. However in the above calculation it does not contribute because of the symmetry of the Riemman tensor.

For a metric satisfying the vacuum Einstein equation globally one can drop the term containing $G_{a b}$ in (3.2). If we integrate the result and apply Stokes' theorem on the left-hand side we get:

$$
(1 / 2) \oint W^{a b} d s_{a b}=\int\left(\nabla_{b} W^{a b}\right) d s_{a}=\int \operatorname{Im}\left(\nabla_{b} \bar{\psi} \gamma^{a b c} \nabla_{c} \psi\right) d s_{a},
$$

where the surface integral is taken over the boundary $\partial \Sigma$ of a four-dimensional spacelike hypersurface $\Sigma$, and where $d s_{a b}$ and $d s_{a}$ are the surface elements of $\partial \Sigma$ and $\Sigma$ respectively. In general the right-hand side of (3.5) is neither positive nor negative. However we can make it to be less than or equal to zero by splitting it into two pieces, one non-positive and one non-negative and then choosing the spinor $\psi$ so as to eliminate the non-negative piece.

For that purpose we adopt the following notation. We use lower case Latin characters starting alphabetically from $i$ to represent an index projected to $\Sigma$. With this convention the last integral in (3.5) can be written as:

$$
\int \operatorname{Im}\left(\nabla_{b} \bar{\psi} \gamma^{a b c} \nabla_{c} \psi\right) d s_{a}=-\int \operatorname{Im}\left(\nabla_{i} \bar{\psi} \gamma^{a} \nabla^{i} \psi\right) d s_{a}+\int \operatorname{Im}\left(\overline{\gamma^{i} \nabla_{i} \psi} \gamma^{a} \gamma^{j} \nabla_{j} \psi\right) d s_{a} .
$$

Here we used the fact that the indices $b, c$ can be replaced by $i, j$ because the index a is contracted with the surface element $d s_{a}$ and $\gamma^{a b c}$ is totally skew, together with the following:

$$
\gamma^{a i j}=\gamma^{a} \gamma^{i j}=\gamma^{a}\left(\gamma^{i} \gamma^{j}-g^{i j}\right)=-\gamma^{i} \gamma^{a} \gamma^{j}-g^{i j} \gamma^{a}=\widetilde{\gamma^{i}} \gamma^{a} \gamma^{j}-g^{i j} \gamma^{a} .
$$

Because $d s_{a}$ is timelike, it follows from the property of the Dirac-adjoint that the first term in (3.6) is non-positive and the second is non-negative. Therefore we conclude that:

$$
(1 / k) \oint W^{a b} d s_{a b} \leqq 0
$$


if $\psi$ satisfies $\gamma^{i} \nabla_{i} \psi=0$ on a spacelike hypersurface $\Sigma$.

b) Connection of (3.5) to Conserved Quantities. In this part we will complete the inequality by interpreting the left-hand side of (3.8) in the limit as the surface $\Sigma$ extends to spatial infinity in terms of conserved quantities.

In the absence of an exact $U(1)$ symmetry we lose the ability to decompose the five-metric into a four-metric, a scalar field, and an electromagnetic field. However, because of our boundary conditions representing an isolated system we have an asymptotic $U(1)$ Killing vector as well as four other asymptotic translation Killing vectors. Then, as in four dimensions, we can construct the total "five-momentum" of the system using these five independent asymptotic Killing vectors. Among its components, the one corresponding to the asymptotic $U(1)$ Killing vector is identified with the total electric charge of the system, while those corresponding to other asymptotic symmetries identified with components of the total fourmomentum of the gravitational field.

In order to relate the surface integral (3.8) with conserved quantities, consider the following expression [9]:

$$
(1 / 2 k) \oint_{\infty}\left(L^{a} \xi^{b}+\Gamma_{m}^{a b} \xi^{m}\right) d s_{a b},
$$

where $L^{a}=\left(\Gamma_{b m}^{a} g^{b m}-\Gamma_{b m}^{b} g^{a m}\right)$, and where $\Gamma_{b c}^{a}$ is defined with respect to the background connection $\stackrel{\circ}{\nabla}_{a}$ by $\Gamma_{a m}^{b} v^{m}=\left(\nabla_{a}-\stackrel{\circ}{\nabla}_{a}\right) v^{b}$. When $\xi^{a}$ is an asymptotically constant vector field ${ }^{2}$ the above integral is ${ }^{5} P_{a} \xi^{a}$, the component of the "fivemomentum" along $\xi^{a}$. Among many possible expressions [9] for the fivemomentum this particular expression turns out to be the most convenient one for our purpose. In a more general setting, or for an asymptotically rotational $\xi^{a}$, one should include [10] an extra term $\stackrel{\circ}{\nabla}^{a} \xi^{b}$ in the integrand of (3.9) and require exact commutativity of $\xi^{a}$ with $\stackrel{\circ}{\nabla}_{a}$ in a neighborhood of spatial infinity. The resulting expression can be written as:

$$
(1 / 2 k) \oint\left(L^{a} \xi^{b}+\nabla^{a} \xi^{b}\right) d s_{a b} .
$$

For the present application, however, the extra term will never contribute, because it reduces to the electric charge of the background, which vanishes as mentioned at the end of the first section.

Let us go back to the spinor expression (3.1) and split the integral of it over a three surface at spatial infinity into two pieces:

$$
\operatorname{Im} \oint_{\infty} \bar{\psi} \gamma^{a b c}\left(\nabla_{c}-\stackrel{\circ}{\nabla}_{c}\right) \psi d s_{a b}+\operatorname{Im} \oint_{\infty} \bar{\psi} \gamma^{a b c} \stackrel{\circ}{\nabla}_{c} \psi d s_{a b} .
$$

Although we know how $\stackrel{\circ}{\nabla}_{a}$ acts on tensors, its extension to spinors is up to us to some extent. Using that freedom we define $\stackrel{\circ}{\nabla}_{a}$ on spinors by (see appendix for further discussion):

$$
\left(\nabla_{a}-\stackrel{\circ}{\nabla}_{a}\right) \psi:=\Gamma_{a} \psi, \quad \Gamma_{a}=(1 / 4) \Gamma_{b c a} \gamma^{b c}
$$

\footnotetext{
2 Because asymptotic Killing vectors are all asymptotically constant any asymptotically constant vector can be regarded as a linear combination of five independent asymptotic Killing vectors
} 
where we used $g_{a b}$ to lower the first index of $\Gamma_{c a}^{b}$. If we substitute this in (3.11) and use the identity (3.4), then we find, first of all, that the first term of (3.11) reduces to $k$ times (3.9) with $\xi^{a}=\operatorname{Im}\left(\bar{\psi} \gamma^{a} \psi\right)$. When $\psi$ is asymptotically constant (we mean that $\left.\psi=O(1), \nabla_{a} \psi=O\left(1 / r^{2}\right)\right)$ its associated vector field $\xi^{a}$ is also asymptotically constant. Thus, for such spinors we conclude that

$$
(1 / k) \operatorname{Im} \oint_{\infty} \bar{\psi} \gamma^{a b c}\left(\nabla_{c}-\stackrel{\circ}{\nabla}_{c}\right) \psi d s_{a b}={ }^{5} P_{a} \xi^{a} .
$$

For the second term of (3.11) we start with the following argument. Although it is not entirely obvious, we claim that (3.11) gives the same answer for any two asymptotically constant spinors differing from each other by $O(1 / r)$. Suppose, in fact, that $\psi_{1}-\psi_{2}=O(1 / r)$. Then

$$
\begin{aligned}
& \operatorname{Im} \oint \bar{\psi}_{1} \gamma^{a b c} \nabla_{c} \psi_{1} d s_{a b}=\operatorname{Im} \oint \bar{\psi}_{2} \gamma^{a b c} \nabla_{c} \psi_{1} d s_{a b} \\
& \quad=-\operatorname{Im} \oint\left(\nabla_{c} \bar{\psi}_{2}\right) \gamma^{a b c} \psi_{1} d s_{a b}=-\operatorname{Im} \oint\left(\nabla_{c} \bar{\psi}_{2}\right) \gamma^{a b c} \psi_{2} d s_{a b} \\
& \quad=\operatorname{Im} \oint \bar{\psi}_{2} \gamma^{a b c} \nabla_{c} \psi_{2} d s_{a b} .
\end{aligned}
$$

In the first and third steps we used $\nabla_{c} \psi_{1}=O\left(1 / r^{2}\right)$ and $\nabla_{c} \psi_{2}=O\left(1 / r^{2}\right)$ respectively, and in the second and fourth steps we used integration by parts. Furthermore, the first term of (3.11) is manifestly insensitive to a change of spinor field by $O(1 / r)$. Hence the second term must be insensitive to those changes, too. This fact will simplify actual evaluation of the second term.

At this point it is interesting to note that in four dimensions, as well as in the trivial topological sector of five-dimensional Kaluza-Klein theory, the second term of (3.11) vanishes while in topologically non-trivial sectors in five-dimensional Kaluza-Klein theory it does not. The reason is simple. In the former cases the appropriate background metrics are flat so that for any asymptotically constant spinor there exists a spinor field with the same asymptotic value which is asymptotically constant with respect to the background. Then according to the above argument the second term will vanish for any asymptotically constant spinor. In the latter cases, however, the background is not flat because of non-vanishing magnetic charge. Consequently, not for all asymptotically constant spinor there is a corresponding strictly covariantly constant spinor with respect to the background, and we cannot discard the second term. In this case it is quite natural for us to expect that the second term, which would have been zero if the topology had been trivial, contains information about magnetic charge of the metric. In fact it is proportional to the magnetic charge.

In order to show that we use the fact (see appendix) that for any given asymptotically constant spinor $\psi$, we can find a spinor $\psi_{P}$ satisfying:

$$
\begin{aligned}
\psi-\psi_{P} & =O(1 / r), \\
\stackrel{\circ}{\nabla}_{a} \psi_{P} & =(1 / 4)\left[(1 / 2) \stackrel{\circ}{F}_{a b} k_{c}-k_{(a} \stackrel{\circ}{F}_{b) c}\right] \gamma^{b c} \psi_{P}+O\left(1 / r^{3}\right) .
\end{aligned}
$$

In the second equation it does not matter which metric is used to raise and lower indices because they agree asymptotically, and $\stackrel{\circ}{F}_{a b}$ is $O\left(1 / r^{2}\right)$. For such a spinor $\psi_{P}$, a short and straightforward calculation, using (3.4) and the fact that the electric 
charge of the background is zero, leads us to:

$$
\begin{aligned}
(1 / k) \operatorname{Im} \oint_{\infty} \bar{\psi}_{p} \gamma^{a b c} \stackrel{\circ}{\nabla}_{c} \psi_{P} d s_{a b} & =\left(\lambda_{\infty} / 4 k\right) \bar{\psi}_{p} \psi_{P} \oint_{\infty}^{*} \stackrel{\circ}{F}^{a b} d \stackrel{\circ}{s}_{a b} \\
& =\left(\pi \lambda_{\infty} / k\right)^{1 / 2} \bar{\psi}_{p} \psi_{P} Q_{M}=(1 / 2 \kappa)^{1 / 2} \bar{\psi}_{p} \psi_{P} Q_{M},
\end{aligned}
$$

where we used the fact that $\bar{\psi}_{P} \psi_{P}$ is asymptotically constant and normalized the magnetic charge by:

$$
Q_{M}=\left(\pi \lambda_{\infty}^{3} / k\right)^{1 / 2}\left(1 / 2 \pi \lambda_{\infty}\right) \oint_{\infty}(1 / 2)^{*} \stackrel{\circ}{F}^{a b} d \stackrel{\circ}{s}_{a b},
$$

where $* \dot{F}^{a b}=(1 / 2) \varepsilon^{a b c d} \stackrel{\circ}{F}_{c d}, \varepsilon^{a} a b c d=\lambda_{\infty}^{-1} \varepsilon^{a b c d e} k_{e}$ and $\dot{F}_{c d}^{\circ}$ is the electromagnetic field of the background metric defined as in Sect. 2. The factor in front of the integral gives $Q_{M}$ the correct dimensions and normalizes it in agreement with the "Heaviside-Lorentz" convention, $\varepsilon_{0}=1$. With this convention, the proportionality constant between the fifth component of five-momentum and electric charge is determined by:

$$
\lambda_{\infty}^{-15} P_{a} k^{a}=\left(\pi \lambda_{\infty} / k\right)^{1 / 2} Q_{E}=(1 / 2 \kappa)^{1 / 2} Q_{E},
$$

where $\kappa=k / 2 \pi \lambda_{\infty}=8 \pi G$ as before. This can be seen most easily by putting $\xi^{a}=k^{a} / \lambda_{\infty}$ in (3.10) and noticing that the first term of the integrand drops out, for $k^{b} d s_{a b}=O(1 / r)$ and $L^{a}=O\left(1 / r^{2}\right)$.

If we add this result to (3.13), (3.8) yields:

$$
\begin{aligned}
(1 / k) \oint_{\infty} W^{a b} d s_{a b} & ={ }^{5} P_{a} \operatorname{Im}\left(\bar{\psi}_{0} \gamma^{a} \psi_{0}\right)+(1 / 2 \kappa)^{1 / 2} \bar{\psi}_{0} \psi_{0} Q_{M}, \\
& \leqq 0
\end{aligned}
$$

provided that for any $\psi_{P}$ satisfying (3.14) we have a solution to $\gamma^{i} \nabla_{i} \psi=0$ which coincides with $\psi_{P}$ asymptotically.

To cast the inequality in its final form we will prove the following. Let $A_{a}$ be a vector and $B$ be a non-vanishing scalar satisfying:

$$
A_{a} \operatorname{Im}\left(\bar{\psi} \gamma^{a} \psi\right) \leqq B \bar{\psi} \psi \quad \text { for all } \quad \psi
$$

Then,

i) $A_{a}$ is a future-directed timelike vector.

ii) $A_{a} A^{a} \leqq-B^{2}$.

First of all note that $\operatorname{Im}\left(\bar{\psi} \gamma^{a} \psi\right)$ is a future-directed timelike vector for any non-zero spinor $\psi$ because of the way the Dirac adjoint is constructed. If we choose a particular $\psi$ such that the right-hand side is negative then the left-hand side will be negative also. Furthermore, it must remain negative under all possible action of the Spin $\uparrow$ group (the double covering of the restricted Lorentz group) because the right-hand side is a scalar. But that is not possible unless $A_{a}$ is itself a future-directed timelike vector, whence i). To prove ii) notice that the above argument implies that

$$
\left|A_{a} \operatorname{Im}\left(\bar{\psi} \gamma^{a} \psi\right)\right| \geqq|B \bar{\psi} \psi|
$$


when $(B \bar{\psi} \psi)$ is negative. When $(B \bar{\psi} \psi)$ is positive (3.20) is still true because if it were violated for some $\psi$ then $\psi^{\prime}:=n_{a} \gamma^{a} \psi$ for any unit vector $n^{a}$ orthogonal to $A_{a}$ would also violate it although $\left(B \bar{\psi}^{\prime} \psi^{\prime}\right)$ is negative, so that we run into a contradiction. Now let us choose for $\psi$ an eigenvector of $A_{a} \gamma^{a}$ with eigenvalue $\lambda$; then (3.20) implies that

$$
|\lambda| \geqq|B|
$$

On the other hand by applying $A_{a} \gamma^{a}$ twice to our eigenvector we obtain $\lambda^{2}=A_{a} A^{a}$. From these ii) trivially follows.

Conversely we claim that if i), ii) are assumed, then (3.19) follows. It suffices to show that

$$
\left|\bar{\psi} \gamma^{0} \psi\right| \geqq|\bar{\psi} \psi| \text { for all } \psi
$$

For this, write $\psi$ as a sum of eigenvectors of $-i \gamma^{0}$, i.e., $\psi=\psi_{+}+\psi_{-}$, where $\psi_{+}=(1 / 2)\left(1-i \gamma^{0}\right) \psi$ and $\psi_{-}=(1 / 2)\left(1+i \gamma^{0}\right) \psi$. Then from the fact that $-i \gamma^{0}$ commutes with Dirac-adjoint, it follows that $\overline{\psi_{+}} \psi_{-}=\overline{\psi_{-}} \psi_{+}=0$. To conclude the proof note that $\left|\bar{\psi} \gamma^{0} \psi\right|=\left|\overline{\psi_{+}} \psi_{+}-\overline{\psi_{-}} \psi_{-}\right|,|\bar{\psi} \psi|=\left|\overline{\psi_{+}} \psi_{+}+\overline{\psi_{-}} \psi_{-}\right|$and $\overline{\psi_{+}} \psi_{+}=$ $\operatorname{Im}\left(\overline{\psi_{+}} \gamma^{0} \psi_{+}\right) \geqq 0, \overline{\psi_{-}} \psi_{-}=-\operatorname{Im}\left(\overline{\psi_{-}} \gamma^{0} \psi_{-}\right) \leqq 0$.

Applying the above result to (3.18) we obtain

$$
\begin{gathered}
-{ }^{5} P_{a}{ }^{5} P^{a}=-{ }^{4} P_{a}{ }^{4} P^{a}-(1 / 2 \kappa) Q_{E}^{2} \geqq(1 / 2 \kappa) Q_{M}^{2}, \\
{ }^{5} P_{a} \text { is timelike and future directed, }
\end{gathered}
$$

or equivalently;

$$
M \geqq\left[(1 / 2 \kappa)\left(Q_{E}^{2}+Q_{M}^{2}\right)\right]^{1 / 2} .
$$

(In non-simplified gravitational and electrical units, we have

$$
\left.M \geqq\left[\left(1 / 16 \pi G \varepsilon_{0}\right)\left(Q_{E}^{2}+Q_{M}^{2}\right)\right]^{1 / 2}\right) .
$$

In the above we assumed that $Q_{M} \neq 0$ for simplicity of argument. However, the inequality (3.21) is still true even if $Q_{M}=0$.

Finally we must prove that for any asymptotically constant spinor $\psi$ there exists a solution $\psi_{w}$ to the equation $\gamma^{i} \nabla_{i} \psi_{w}=0$ such that $\psi_{w}$ agrees with $\psi$ asymptotically. Fortunately proofs already exist in the literature [11] in the case of four dimensions and the basic inequality ${ }^{3}$ still holds in our case.

\section{Conclusion}

We proved that any globally regular solution to the five-dimensional vacuum Einstein equation satisfying Kaluza-Klein boundary conditions should satisfy the inequality (3.21). In each asymptotic topological sector characterized by magnetic charge $Q_{M}$, the minimum energy is achieved when $Q_{E}$ is zero. In the trivial

\footnotetext{
3 In the first paper of ref. [11] it is called the "generalized Hardy lemma." In the second paper, a
} slightly more general version of it is proved in the appendix 
topological sector $\left(Q_{M}=0\right)$ the minimum energy is zero and it is of course attained by the Kaluza-Klein vacuum solution. In non-trivial topological sector $\left(Q_{M} \neq 0\right)$ the multi-monopole solutions have precisely the minimum energy. With normalization, the mass, electric charge and magnetic charge of the multi-monopole solutions are

$$
M=n \ell(1 / 2 \kappa), \quad Q_{E}=0, \quad Q_{M}=n \ell(1 / 2 \kappa)^{1 / 2}
$$

respectively, where $\ell:=2 \pi \lambda_{\infty}$ and $n$ is the number of monopoles; this clearly saturates the inequality (3.21). Thus our results strongly support stability of the multi-monopole solutions even against non- $U(1)$-symmetric perturbations of finite size.

The inequality (3.21) is weaker than the corresponding one in four-dimensional Einstein-Maxwell theory by a factor of two as pointed out in [5]. Nevertheless, this result is the optimal one since as just pointed out there exist solutions saturating the inequality.

Of course one can "dimensionally reduce" to obtain an equivalent fourdimensional configuration using the $U(1)$ symmetry and ask why the ordinary four-dimensional inequality is violated. One obvious reason for that would be the presence of a scalar field of Brans-Dicke type and the attractive nature of it. It would be interesting to understand more on the role of the scalar field ${ }^{4}$.

\section{Appendix}

In Sect. 3 we extended the connection $\stackrel{\circ}{\nabla}_{a}$ associated with the background metric $\stackrel{\circ}{g}_{a b}$ to spinor fields by:

$$
\left(\nabla_{a}-\stackrel{\circ}{\nabla}_{a}\right) \psi=(1 / 4) \Gamma_{b c a} \gamma^{b c} \psi .
$$

On the other hand, suppose we defined $\gamma^{a}:=S_{m}^{a} \gamma^{m}$ for an invertible mapping $S_{b}^{a}$ satisfying $\dot{g}^{a b}=S_{m}^{a} S_{n}^{b} g^{m n}$ and $S_{b}^{a}=\delta_{b}^{a}+O(1 / r)$. In this case it would be natural to extend the background connection to spinors by demanding the following condition:

$$
\dot{D}_{a} \gamma^{o b}=0 .
$$

Here, we have used $\dot{D}_{a}$ for the second extension to distinguish it from the first. How are these two extensions related to each other? Let us show that their difference can be made to be $O\left(1 / r^{3}\right)$ by choosing an appropriate $S_{b}^{a}$. Let

$$
\left(\stackrel{\circ}{\nabla}_{a}-\stackrel{\circ}{D}_{a}\right) \psi=(1 / 4) C_{m n a} \gamma^{m n} \psi, \quad C_{m n a}=-C_{n m a} .
$$

Then

$$
\stackrel{\circ}{\nabla}_{a} \hat{\gamma}^{b}=\left(\stackrel{\circ}{\nabla}_{a}-\stackrel{\circ}{D}_{a}\right) \hat{\gamma}^{b}=(1 / 4) C_{m n a} S_{c}^{b}\left[\gamma^{m n}, \gamma^{c}\right]=C_{m n a} S_{c}^{b} \gamma^{m} g^{n c},
$$

4 A different treatment of the scalar field seems to account for the discrepancy between our inequality (3.21) and the one given in the second paper of ref. [4]. An extra term containing the scalar field in that paper is absorbed into the mass term when the natural conformal factor for the reduced four-metric is chosen 
where we have used (A.2) and the fact that two derivative operators $\stackrel{\circ}{\nabla}_{a}$ and $\dot{D}_{a}$ agree on spacetime tensors. On the other hand, using (A.1) we obtain:

$$
\begin{aligned}
\stackrel{\circ}{\nabla}_{a} \gamma^{m} & =\left(\nabla_{a}-\dot{\nabla}_{a}\right) \gamma^{m}=-\Gamma_{a c}^{m} \gamma^{c}-(1 / 4) \Gamma_{c n a}\left[\gamma^{c n}, \gamma^{m}\right] \\
& =-\Gamma_{a c}^{m} \gamma^{c}-\Gamma_{c n a} \gamma^{[c} g^{n] m}=-g^{m n}\left(\Gamma_{n a c}+\Gamma_{[c n] a}\right) \gamma^{c} \\
& =-(1 / 2) g^{m n} \Gamma_{(c n) a} \gamma^{c}=-(1 / 2) g^{m n}\left(\stackrel{\circ}{\nabla}_{a} g_{n c}\right) \gamma^{c} .
\end{aligned}
$$

Thus,

$$
\stackrel{\circ}{\nabla}_{a} \hat{\gamma}^{b}=\left(\stackrel{\circ}{\nabla}_{a} S_{m}^{b}\right) \gamma^{m}+S_{m}^{b} \stackrel{\circ}{\nabla}_{a} \gamma^{m}=\left(\stackrel{\circ}{\nabla}_{a} S_{m}^{b}\right) \gamma^{m}-(1 / 2) S_{m}^{b} g^{m n}\left(\stackrel{\circ}{\nabla}_{a} g_{n c}\right) \gamma^{c} .
$$

Multiplying both right-hand sides of (A.3) and (A.5) by $\stackrel{\circ}{d b}_{d} S_{e}^{d}$ we get:

$$
\begin{aligned}
C_{m e a} \gamma^{m} & =\stackrel{\circ}{g}_{d b} S_{e}^{d} \stackrel{\circ}{\nabla}_{a} \hat{\gamma}^{b}=\left[\stackrel{\circ}{g}_{d b} S_{e}^{d} \stackrel{\circ}{\nabla}_{a} S_{m}^{b}-(1 / 2) \stackrel{\circ}{\nabla}_{a} g_{e m}\right] \gamma^{m} \\
& =\left[\stackrel{\circ}{g}_{d b} S_{[e}^{d} \stackrel{\circ}{\nabla}_{|a|} S_{m]}^{b}\right] \gamma^{m},
\end{aligned}
$$

where we used $g_{e m}=\stackrel{\circ}{g}_{d b} S_{e}^{d} S_{m}^{b}$. Drop $\gamma^{m}$ from both sides, then

$$
C_{m e a}=\left[\stackrel{\circ}{g}_{d b} S_{[e}^{d} \stackrel{\circ}{\nabla}_{|a|} S_{m]}^{b}\right] .
$$

Now let us write $S_{m}^{b}=\delta_{m}^{b}+T_{m}^{b}+O\left(1 / r^{2}\right)$ and make $\stackrel{\circ}{g}_{b e} T_{m}^{b}$ symmetric using gauge freedom. Then, with such a choice of $S_{m}^{b}$ it immediately follows that $C_{a n m}$ is $O\left(1 / r^{3}\right)$ since $T_{m}^{b}$ is $O(1 / r)$ and $\stackrel{\circ}{\nabla}_{a} T_{m}^{b}$ is $O\left(1 / r^{2}\right)$. Therefore, $\stackrel{\circ}{a}_{a}$ and $\dot{D}_{a}^{\circ}$ agree to $O\left(1 / r^{3}\right)$.

With this result in mind let us go back to Eq. (3.14). Any solution $\psi_{P}$ will satisfy

$$
\dot{\circ}_{a} \psi_{P}=(1 / 4)\left[(1 / 2){\stackrel{\circ}{F_{a b}}}_{k} k_{c}-k_{(a} \stackrel{\circ}{F}_{b) c}\right] \gamma^{\circ b c} \psi_{P}+O\left(1 / r^{3}\right)
$$

and vice versa. Now, we will consider (A.7) and the background $\left(\dot{g}_{a b}, \dot{\gamma}^{a}\right)$ instead of (3.14) and $\left(g_{a b}, \gamma^{a}\right)$. Tensor indices will be raised and lowered by the background metric.

To understand this equation introduce the following orthonormal basis:

$E_{0}^{a}$ is chosen to be the normalized static Killing vector,

$E_{5}^{a}=\lambda_{\infty}^{-1} k^{a}$,

$E_{1}^{a}, E_{2}^{a}, E_{3}^{a}$ are chosen in such a way that these are mapped under the projection mapping into normalized spatial translational Killing vectors in the flat quotient manifold.

If we define

$$
\stackrel{\circ}{D}_{a} E_{i}^{b}=\omega_{a c}^{b} E_{i}^{c}, \quad i=0,1,2,3,5,
$$

we can easily verify that the expression inside the bracket of (A.3) is exactly $\omega_{a b c}$. Now recall that in terms of an orthonormal basis

$$
\stackrel{\circ}{D}_{a} \psi=E_{a} \psi+(1 / 4) \omega_{a b c} \gamma^{\circ b c} \psi,
$$

where $E_{a} \psi$ is meant to be the Pfaff derivative of $\psi$ (i.e., the spinor whose components with respect to the basis $E_{i}^{a}$ are derivative of the components of $\psi$ with respect to the same basis). Thus, Eq. (3.14) or equivalently (A.7) can be solved trivially if we choose a spinor whose components are constant with respect to the basis $E_{i}^{a}$. Finally, because our orthonormal basis is asymptotically constant any asympto- 
tically constant spinor should have constant components with respect to our basis up to the $O(1 / r)$ term.

Acknowledgements. We would like to thank one of the referees for suggesting ways to improve the presentation of this paper.

\section{References}

1. Witten, E.: A new proof of the positive energy theorem. Commun. Math. Phys. 80, 381-402 (1981)

2. Schoen, R., Yau, S.-T.: On the proof of the positive mass conjecture in general relativity. Commun. Math. Phys. 65, 45-76 (1979); Proof of the positive mass theorem II. Commun. Math. Phys. 79, 231-260 (1981)

3. Nester, J. M.: A new gravitational expression with a simple positivity proof. Phys. Lett. 83A, 241-242 (1981); Nester, J. M., Israel, W.: Positivity of Bondi gravitational mass. Phys. Lett. 85A, 259-260 (1981); Gibbons, G. W., Hawking, S. W., Horowitz, G. T., Perry, M. J.: Positive energy theorems for black holes. Commun. Math. Phys. 88, 295-308 (1983); Gibbons, G. W., Hull, C. M.: A Bogomolny bound for general relativity and solutions in $N=2$ supergravity. Phys. Lett. 109B, 190-194 (1982); Abbott, G., Deser, S.: Stability of the gravity with a cosmological constant. Nucl. Phys. B195, 76-96 (1982)

4. Moreschi, O. M., Sparling, G. A. J.: On the formulation of the positivity-energy theorem in Kaluza-Klein theories. J. Math. Phys. 27 (9), 2402-2408 (1986); Taub, A. H.: Positivity of energy in five-dimensional classical unified field theories. Lett. Math. Phys. 9, 243-253 (1985)

5. Gibbons, G. W., Perry, M. J.: Soliton supermultiplets and Kaluza-Klein theory. Nucl. Phys. B248, 629-646 (1984)

6. Mecklenburg, W.: The Kaluza-Klein Idea. Status and Prospects. Fortschr. Phys. 32 (5), 207-260 (1984); Lee, C. H.: An introduction to Kaluza-Klein theories. Singapore: World Scientific 1984

7. Sorkin, R. D.: Kaluza-Klein monopole. Phys. Rev. Lett. 51 (2), 87-90 (1983)

8. Goldberg, J. N.: Invariant transformations, conservation laws, and energy-momentum. In: General relativity and gravitation, Vol. 1. Held, A. (ed.). New York: Plenum Press 1980

9. Bombelli, L., Koul, K., Kunstatter, G., Lee, J., Sorkin, R. D.: On energy in five-dimensional gravity and the mass of the Kaluza-Klein monopole. (to appear)

10. Sorkin, R. D.: Conserved quantities as action variations. Proc. Conf. on Mathematics in General Relativity, Santa Cruz, June 1986 (to appear)

11. Reula, O., Tod, K. P.: Positivity of Bondi energy. J. Math. Phys. 25 (4), 1004-1008 (1984); Parker, T. H.: Gauge choice in Witten's energy expression. Commun. Math. Phys. 100, 471-480 (1985)

Communicated by S.-T. Yau

Received December 29, 1986; in revised form August 8, 1987 